\title{
Study on the High Energy Particle Detector calorimeter
}

\section{Beatrice Panico*}

INFN Napoli, I-80126 Naples, Italy

E-mail: bpanico@na.infn. it

G. Osteria, F. Perfetto, V. Scotti

INFN Napoli, I-80126 Naples, Italy

P. Cipollone, C. De Donato, C. De Santis, L. Marcelli, G. Masciantonio, M. Mergè, F. Palma, P. Picozza, A. Sotgiu, R. Sparvoli

INFN, Sezione di Roma Tor Vergata, I-00173 Rome, Italy

A. Contin, C. Guadalini, G. Laurenti, M. Lolli, F. Palmonari, L. Patrizii, M. Pozzato INFN, Sezione di Bologna, I-40127 Bologna, Italy

M. Ricci, B. Spataro

INFN, Frascati National Laboratories, I-00044 Frascati - RM, Italy

W.J. Burger, R. Battiston, F.M. Follega, R. luppa, I. Lazzizzera, C. Manea, I. Rashevskaya, E. Ricci, V. Vitale,

INFN, Sezione di Trento, I-38123 Povo - TN, Italy

G. Ambrosi, M. Ionica, M. Sposito

INFN Perugia, I-06123 Perugia, Italy

\section{S. Bartocci, L. Conti}

International Telematic University UNINETTUNO, I-00186 Roma, Italy

G. Castellini, L. Pacini, S.B. Ricciarini

IFAC-CNR, I-50019 Sesto Fiorentino - FI, Italy 
The High-Energy Particle Detector (HEPD) is one of the payloads of the CSES space mission, built by the Italian 'Limadou' collaboration. It will study the temporal stability of the inner Van Allen radiation belts, the precipitation of trapped particles in the atmosphere and the low energy component of the cosmic rays (5 - $100 \mathrm{MeV}$ for electrons and $10-300 \mathrm{MeV}$ for protons). Results of the test beams held at the Beam Test Facility of the INFN National Laboratory of Frascati, for electrons, and at the Proton Cyclotron of Trento, for protons, will be presented. The performance of the apparatus both on the energy reconstruction and in the lepton/hadron separation will be shown.

35th International Cosmic Ray Conference - ICRC2017

10-20 July, 2017

Bexco, Busan, Korea

* Speaker. 


\section{The High-Energy Particle Detector}

The High-Energy Particle Detector (HEPD) is one of the payloads of the CSES space mission, composed by several instruments. This is a space mission with different goals spreading from the measurement of signals from electromagnetic emission in ionosphere to the measurement of background magnetic field in space, to the cosmic ray measurements in an energy range up to few hundreds of $\mathrm{MeV}$ [1]. The satellite will be placed in a 97,4 $4^{\circ}$ inclination Sun-synchronous circular orbit, at an altitude of $\sim 500 \mathrm{~km}$.

The HEPD is developed to study protons and electrons in the energy range [3-200] MeV [2]. The main requirements are a good particle identification and energy resolution, as is reported in Table 1.

\begin{tabular}{ll}
\hline Parameter & Value \\
\hline Energy Range & Electrons: 3-100 MeV \\
Energy Range & Protons: $30-200 \mathrm{MeV}$ \\
Angular resolution & $<8^{\circ} @ 5 \mathrm{MeV}$ \\
Energy resolution & $<10 \% @ 5 \mathrm{MeV}$ \\
Particle identification & $>90 \%$ \\
Free field of view & $\geq 70^{\circ}$ \\
Pointing & Zenith \\
Operative temperature & $-10^{\circ} ;+45^{\circ}$ \\
\hline
\end{tabular}

Table 1: HEPD main technical characteristics.

To study the low energy component of the cosmic rays, the HEPD detector is composed by different instruments [3]:

- a tracker, made of two planes of double-side silicon micro-strip sensors, at the top of the instrument, to provide the direction of the incident particle;

- the trigger system, which consists of two layers of plastic scintillators, the upper thin one is divided into 6 segments, while the second is the first calorimeter plane;

- a calorimeter, composed by 16 plastic scintillators with dimensions $(15 \times 15 \times 1) \mathrm{cm}^{3}$ and a layer of 9 LYSO cubes, for a resulting plane of dimension $(15 \times 15 \times 4) \mathrm{cm}^{3}$;

- a scintillator veto system, $5 \mathrm{~mm}$ thick, at sides and at the bottom of the instrument.

\section{MC Simulation}

A Monte Carlo simulation based on Geant 4 toolkit was developed in order to evaluate the HEPD performances [4]. In Fig. 1 different views of the simulated detectors have been showed. In Fig. 1A the HEPD detectors and the electronic box are showed.

In Fig. 1B the 16 scintillator planes are showed; for each plane the signal is read by 2 PMTs. It is also visible the structure which contains the scintillators.

In Fig. $1 \mathrm{C}$ all the HEPD detectors are showed. From the left: 


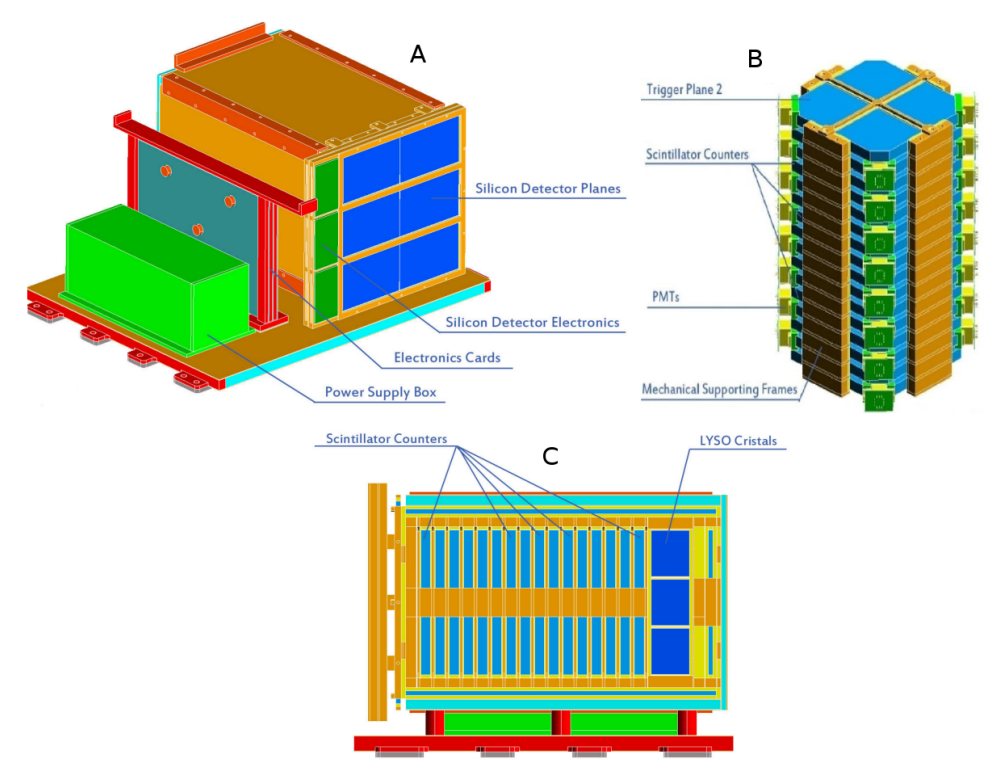

Figure 1: View of the HEPD electric box and detectors.

- the silicon plane (orange);

- the 16 planes (light blue);

- the lyso crystals (blue);

- the veto system surrounding the calo (yellow).

In order to estimate the performances of HEPD, $10^{9}$ events have been uniformly generated according to a power law spectrum $E^{-\gamma}$, where:

- $\gamma=2.2$, for electrons in the energy range [1-200] MeV;

- $\gamma=2.7$, for protons in the energy range [10-500] MeV;

Events have been generated starting from a spherical surface around HEPD to ensure an uniform exposure for the detector.

The simulation played an important role for the choice of the trigger mask. During its orbit HEPD crosses various orbital zones where there is a different rate of cosmic rays. To avoid the instrument saturation, 8 trigger masks have been implemented. The basic one provides the coincidence between the trigger plane (T1) and the first calorimeter plane (P1). For zones with a greater cosmic ray flux the mask can be changed requiring the coincidence of the signals given by other calorimeter planes. In Fig. 2 the trigger rate is reported setting the basic trigger for electrons and protons (blue line). The red line represents the trigger rate for those events without a signal into the lateral veto; the black line represents the trigger rate for events completely contained into the detector. In Fig. 3 a different trigger mask has been set; it requires the coincidence of the trigger plane (T1), of the first (P1) and second (P2) calorimeter plane. 

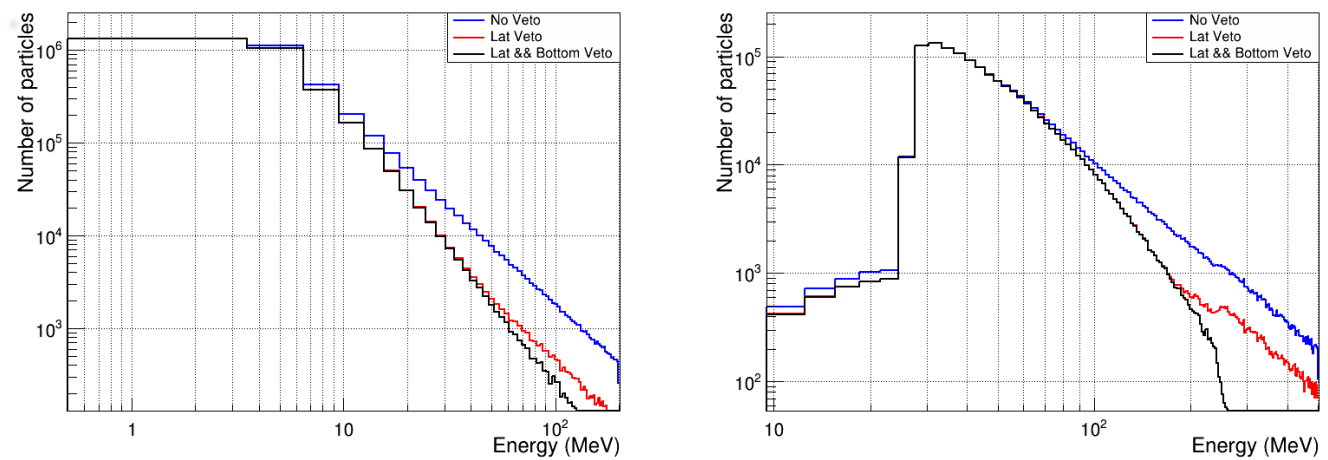

Figure 2: Event rate obtained with the trigger basic mask (T1 \&\& P1) for simulated electrons (left) and protons (right). The blue line represents all detected events; the red line represents events without a signal into the lateral veto; the black line represents events completely contained into the detector.
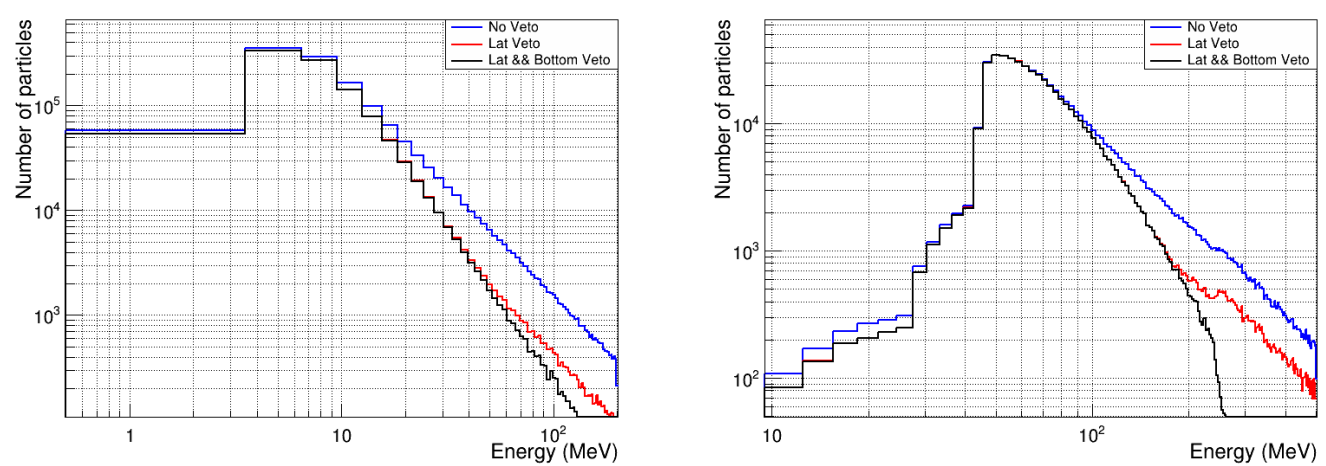

Figure 3: Event rate obtained with the trigger mask (T1 \&\& P1 \&\& P2) for simulated electrons (left) and protons (right). The blue line represents all detected events; the red line represents events without a signal into the lateral veto; the black line represents events completely contained into the detector.

\subsection{Energy resolution}

The HEPD calorimeter can be divided into two parts: the upper calorimeter, made by plastic scintillators, and the lower calorimeter, made by Lyso cristals. To study the energy resolution of the detector, samples of protons and electrons at fixed energies have been simulated. The simulated energy range is [3-150] $\mathrm{MeV}$ for electrons and [30-200] $\mathrm{MeV}$ for protons.

In Fig. 4 the energy deposited in each detector layer is reported for electron beams of different initial energy.

In Fig. 5(left) the energy losses of electrons with energy $\mathrm{E}=100 \mathrm{MeV}$ into the upper and lower calorimeter are reported. The green line represents the total energy released by the particle; the magenta line represents the energy deposited into the upper calorimeter, while the blue line is the energy released into the Lyso cristals. In Fig. 5(right) the profile of the energy released into the upper calorimeter vs the lower calorimeter (Lyso) is reported. In Fig. 6 the same plots are reported for a beam of protons with energy $\mathrm{E}=200 \mathrm{MeV}$. 


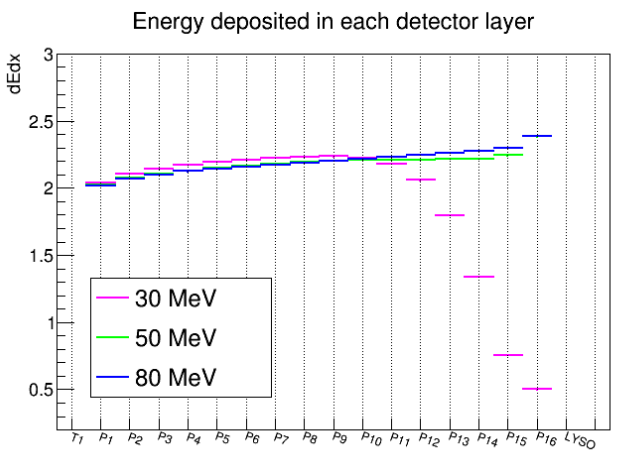

Figure 4: Profile of the energy released by electrons with different energies into the calorimeter for each layer.
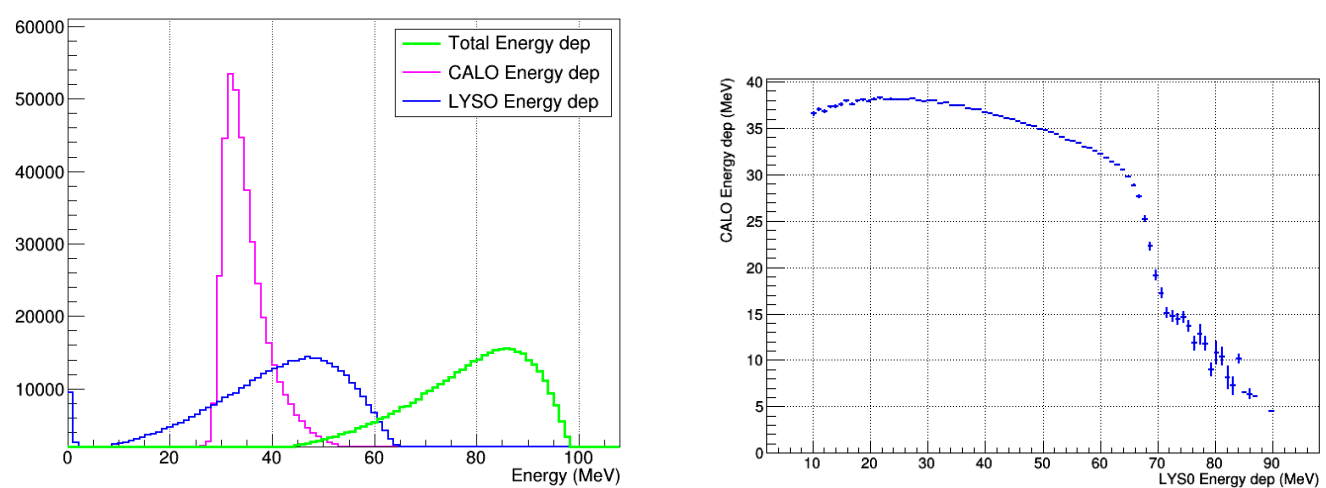

Figure 5: Energy losses of electrons with initial energy $\mathrm{E}=100 \mathrm{MeV}$ into the upper and lower calorimeter (left). Profile of the energy released into the upper calorimeter vs the lower calorimeter (right).

In Fig. 7 the energy resolution of the HEPD calorimeter is reported. Only events completely contained into the calorimeter have been considered. The energy resolution has to be $<10 \%$ at 5 $\mathrm{MeV}$. For both electrons and protons results are in good agreement with these initial requests. The
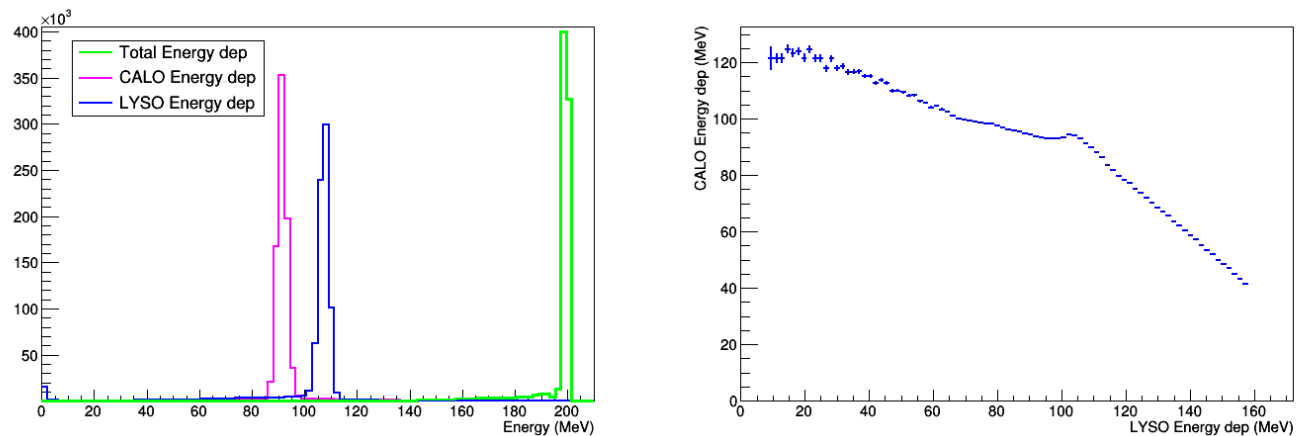

Figure 6: Energy losses of protons with energy $\mathrm{P}=200 \mathrm{MeV}$ into the upper and lower calorimeter (left). Profile of the energy released into the upper calorimeter vs the lower calorimeter (right). 

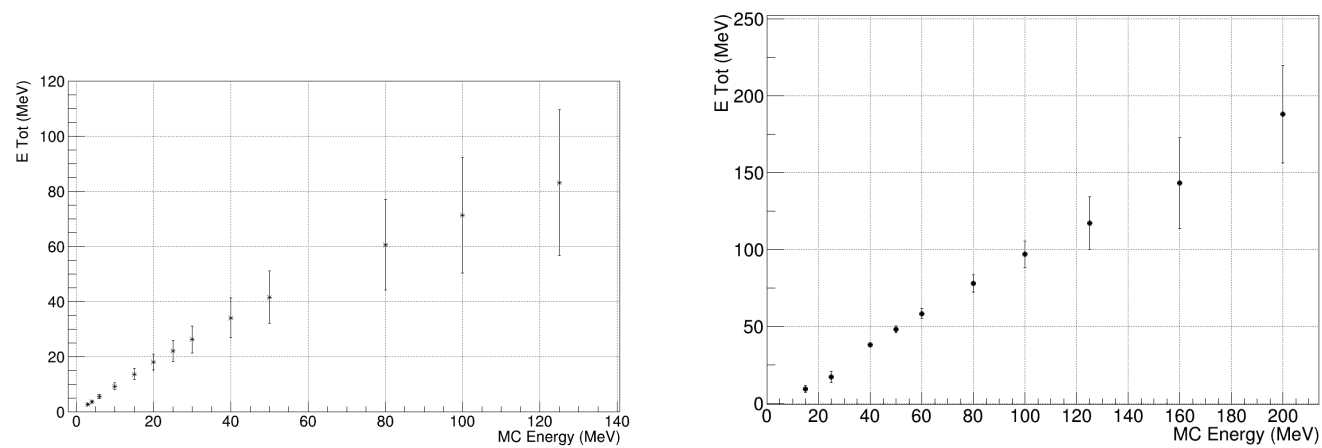

Figure 7: Energy resolution for the calorimeter for electrons (left) and protons (right).

energy losses inside the material of the mechanical structures are responsible of the low energy tails observed in the distributions. Due to the LYSO crystals at the end of the calorimeter, protons are fully contained for energies between [150 - 250] MeV.

\section{Test beams}

The HEPD project involved the construction of 4 detector versions: the Electrical Model (EM), the Structural and Thermal Model, the Qualification Model (QM) and the Flight Model (FM). In May 2017, The FM was tested at the Beam Test Facility (BTF) of the 'Laboratori Nazionali di Frascati' of INFN with an electron/positron beam with energy in the range [30-120] MeV [5].

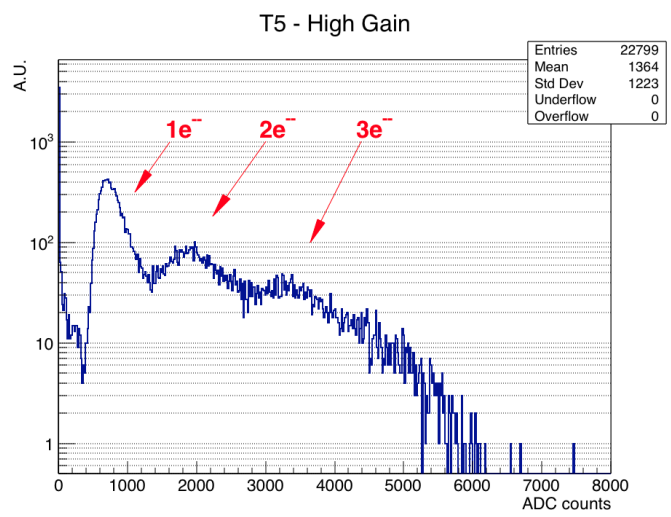

Figure 8: Energy released by electrons with $30 \mathrm{MeV}$ on one paddle of the trigger plane.

In Fig. 8 the numbers of counts on the trigger paddle hit by the beam has been showed. The signal induced by 1,2 , and 3 contemporary particles can be clearly seen. The counts as a function of the plane of the upper calorimeter has been showed in Fig. 9 for electrons with energy of 120 $\mathrm{MeV}$. The signal is coherent with the expectations; as reported in Fig. 4, electrons have a constant energy loss for energy greater than $50 \mathrm{MeV}$. The correlation between test beam data and simulation results allows the calibration of the instrument with a good precision. 


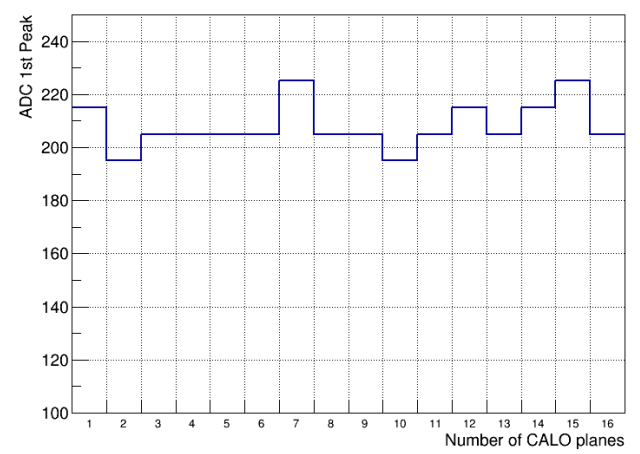

Figure 9: Energy released by electrons with $120 \mathrm{MeV}$ on the single layers of the calorimeter.

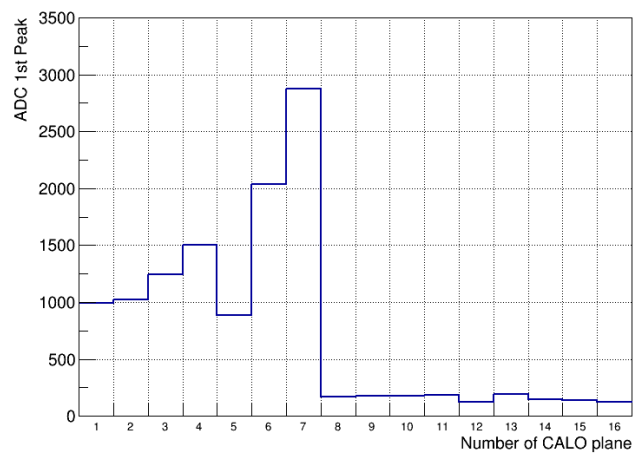

Figure 10: Energy released by protons with $100 \mathrm{MeV}$ on the single layers of the calorimeter.

In October 2017, the FM was tested at the Proton Cyclotron of Trento with proton beams with energy included into the range [70-200] MeV. In Fig. 10 the signal detected as a function of the single layers of the calorimeter is reported for a proton beam with an initial energy of $100 \mathrm{MeV}$.

\section{Conclusions}

In this paper the performances of the HEPD detector, a payload mounted on the CSES satellite, have been described. We would like to thank the Beam Test Facility at 'Laboratori Nazionali di Frascati' and the Proton Cyclotron of Trento for the possibility to test the detector. In this paper some results obtained for HEPD at the test beams are reported. More data are under study.

\section{References}

[1] Wang L. et al., Earthq Sci (2015) 28 4, 303

[2] Picozza P. et al., Proceedings of the 35th ICRC, Busan, South Corea

[3] Sparvoli et al., Proceedings of the Living Planet Symposium 2016, Prague, Czech Republic

[4] Panico B. et al., Nuclear and Particle Physics Proceedings, DOI:1016/j.nuclphysbps.2017.06.023

[5] Ghigo A. et al., (2003) Nucl.Instrum.Meth. A 515, 524 